\title{
Electron emission in ionization of He and Ne by fast dressed oxygen ions and projectile-charge-state dependence
}

\author{
Shubhadeep Biswas, ${ }^{1, *}$ S. Kasthurirangan, ${ }^{1,2}$ D. Misra, ${ }^{1}$ J. M. Monti, ${ }^{3}$ R. D. Rivarola, ${ }^{3}$ P. D. Fainstein, ${ }^{4}$ and L. C. Tribedi ${ }^{1, \dagger}$ \\ ${ }^{1}$ Tata Institute of Fundamental Research, Homi Bhabha Road, Colaba, Mumbai 400 005, India \\ ${ }^{2}$ Institute of Chemical Technology, Matunga, Mumbai-400019, India \\ ${ }^{3}$ Institute de Fisica Rosario (CONICET-UNR), Universidad Nacional de Rosario, 2000 Rosario, Argentina \\ ${ }^{4}$ Comision Nacional de Energa Atomica, Centro Atomico Bariloche, 8400 San Carlos de Bariloche (RN), Argentina
}

(Received 18 February 2014; revised manuscript received 24 October 2014; published 26 February 2015)

\begin{abstract}
The double-differential cross sections (DDCS) of low-energy electrons emitted at forward, backward, and perpendicular directions are reported for collisions of $3.75 \mathrm{MeV} / \mathrm{u} \mathrm{O}^{q+}(q=5,6,7,8)$ projectiles with He and Ne targets. The measured DDCS are found to be deviating from the $q^{2}$ dependence throughout the entire energy region. The effect of projectile electrons, for the dressed ions, as a function of the impact parameter is clearly noticeable for large as well as low-impact parameter collisions. We also present a theoretical calculation based on the prior form of the continuum distorted wave-eikonal initial state approximation, in which the projectile-active electron interaction is modeled with the Green-Sellin-Zachor potential. This particular representation of the potential has been proven to give good qualitative results for projectiles with residual electrons. In addition to the total DDCS, the individual contributions from target ionization, projectile electron loss, and simultaneous ionization processes are also calculated. The total DDCS obtained from these calculations are shown to be in excellent agreement with the experimental observations.
\end{abstract}

DOI: 10.1103/PhysRevA.91.022711

PACS number(s): $34.50 . \mathrm{Fa}, 34.50 . \mathrm{Gb}$

\section{INTRODUCTION}

In energetic ion-atom collisions, production of the lowenergy electrons in target ionization is a result of mutual Coulomb interactions among the residual target, the active electron, and the ionic projectile [1]. The cross section of electron production is directly dependent on the projectile charge state $(q)$ and the impact parameter of the collision. Most of the earlier studies have been carried out with bare ions as the projectile, for which $q$ is the same as the projectile atomic number $\left(Z_{p}\right)[1-7]$. But relatively few examples are found in the literature which involve dressed ion projectiles. In the case of such dressed ion projectiles, the situation is more complicated because of the additional interactions between the projectile electrons and the active electron. The effect of these electrons can differ depending on the impact parameter. For large enough impact parameters, the projectile electrons only participate passively by screening the projectile nuclear charge. However, as the impact parameter decreases, the projectile electrons intervene actively in the collision, increasing the ionization cross section $[1,8]$.

The $Z_{p}$ dependence as well as the $q$ dependence of the total ionization cross section (TCS) for different atomic and molecular targets have been a subject of extensive study for a long time [9-13]. Moreover, the $Z_{p}$-dependence study in the double-differential cross section (DDCS) level, involving only bare ions as the projectile, has also been performed in numerous cases [14-18]. These studies revealed important information regarding the applicability of the Born approximation and the role of the two-center effect on electron emission. In the 1990s, similar investigations for the binary encounter (BE) process became appealing in the study of ion-atom

\footnotetext{
*shubhadeep@tifr.res.in

†lokesh@tifr.res.in
}

ionization. Some experiments showed perfect $Z_{p}^{2}$ dependence (Born scaling) with a wide range of bare ion projectiles [19]. On the other hand, some other experimental and theoretical studies involving dressed projectiles revealed so-called anomalous scaling behavior in the BE electron emission spectrum [20-25]. In apparent contradiction to the expected Born scaling (i.e., $q^{2}$ ) of DDCS, an inverse $q$ dependence was seen for BE electron emission. An interference pattern superimposed on the $\mathrm{BE}$ peak for $1 \mathrm{MeV} / \mathrm{u} \mathrm{U}^{21+}$ impinging on $\mathrm{He}$ [26] was also observed. All of these apparently unusual features were explained on the basis of the effect of the projectile electrons on the close impact parameter target ionization process. At the same point of time, some other studies addressed the problem of the target-projectile electron correlation effect. Those studies showed that apart from the one-electron processes of single excitation and single ionization, two-electron processes, such as transfer excitation and transfer ionization, can take place due to the interaction between the target electron and the projectile electron. This kind of correlation effect is enhanced with the decreasing projectile energy [27-33]. In this regard, several theoretical pictures were also developed starting from the pioneering work by Bates-Griffing [34-37]. Very recently, the absolute TCS measurements along with the continuum distorted wave-eikonal initial state (CDW-EIS) calculation by Wolff et al. have demonstrated that the production of highly charged recoils is strongly enhanced in the case of a dressed projectile when compared to the bare projectile with the same charge state, at the same velocity [38]. Therefore, it is clearly understood that the interaction mechanism in the case of dressed ion projectiles, which eventually turns out to be the simultaneous many-body interaction, is considerably different than that of the bare ion projectiles. As a result, proper systematic experimental and theoretical investigations with dressed projectiles are needed to explore this subject in detail. 
In this regard, the study of electron DDCS can add useful input to the understanding of the effect of projectile electrons on different ionization processes, which are identifiable in a typical DDCS spectrum. However, the $q$-dependence study in the DDCS level, involving dressed projectiles, is relatively scarce in the literature. In particular, few measurements [25,39-45] were carried out in the low-electron energy region. These above-mentioned measurements were interpreted qualitatively by introducing an important idea of projectile screening. But, most of the cases were lacking a proper $a b$ initio theoretical calculation which could explain the entire spectrum. These measurements were also mainly restricted to the extreme forward angles. Other than that, there are few measurements available which involve low- $Z$ atoms as the projectile $[46,47]$. Therefore, because of a lack of systematic experimental data and proper theoretical calculations covering a wide energy and angular range, the influence of projectile electrons in the low-energy region is still to be understood in detail.

Here we report the absolute DDCS measurements of low-energy electron emission from the collisions of fast $\mathrm{O}$ ions $(q=5,6,7,8)$ with $\mathrm{He}$ and Ne targets, at forward, backward, and intermediate angles. We have also carried out theoretical calculations based on the Green-Sellin-Zachor (GSZ) active electron-projectile interaction model potential [48] in the prior version [49] of the CDW-EIS framework $[15,50,51]$, as prescribed recently in Refs. [52,53]. In addition to the total DDCS, the contributions from different ionization processes, relevant for the dressed projectile impact, are also estimated. For reference, it should be mentioned here that some of the contributions were estimated earlier using the Born approximation [54-58], classical trajectory Monte Carlo method (CTMC) [59], or binary encounter approximation [60]. Reference [59] also includes the CDW calculation, including the effective projectile charge to describe the interaction. But it is instructive to carry out calculations using the CDW-EIS model, which has been proven to be one of the successful models for the fast highly charged projectiles. In this respect, the present study enables us to give stringent verification to the present theoretical model, which is specifically developed for dressed projectiles treating the influence of projectile electrons separately, not in an average manner. It also helps to make a comparison between different targets such as $\mathrm{He}$ and $\mathrm{Ne}$. Apart from that, this study demonstrates the deviation of $q$ dependence from Born scaling at the DDCS level, which is solely caused by the existence of electrons in the projectile. By virtue of the data for different charge states, we have tried to scale the low-energy part of the spectrum by a $q^{n}$ kind of dependence and the angle-dependent values of $n$ have also been given in tabular form (in Table I) for ready reference.

TABLE I. Values of $n$ in $q^{n}$ at different angles obtained from the experiment and CDW-EIS (theory) calculation for the Ne. Associated errors, due to fitting, are displayed side by side.

\begin{tabular}{llr}
\hline \hline Angle & Experiment & Theory \\
\hline $30^{\circ}$ & $1.38 \pm 0.10$ & $1.33 \pm 0.18$ \\
$90^{\circ}$ & $1.29 \pm 0.10$ & $1.20 \pm 0.18$ \\
$150^{\circ}$ & $1.12 \pm 0.10$ & $1.04 \pm 0.16$ \\
\hline \hline
\end{tabular}

TABLE II. Measured double-differential cross sections for He in units of $\mathrm{Mb} /(\mathrm{eV} \mathrm{sr})$ at different angles, for different charge states. For errors, see the text. Numerals in square brackets denote the power of 10.

\begin{tabular}{lcccccc}
\hline \hline & 30 & $30^{\circ}$ & $90^{\circ}$ & $90^{\circ}$ & $150^{\circ}$ & $150^{\circ}$ \\
$\epsilon_{e}(\mathrm{eV})$ & $\mathrm{O}^{5+}$ & $\mathrm{O}^{8+}$ & $\mathrm{O}^{5+}$ & $\mathrm{O}^{8+}$ & $\mathrm{O}^{5+}$ & $\mathrm{O}^{8+}$ \\
\hline 1 & $1.11[0]$ & $2.56[0]$ & $1.36[0]$ & $2.85[0]$ & $7.00[-1]$ & $1.17[0]$ \\
5 & $6.45[-1]$ & $1.49[0]$ & $8.23[-1]$ & $1.70[0]$ & $4.12[-1]$ & $6.97[-1]$ \\
9 & $4.61[-1]$ & $1.06[0]$ & $5.92[-1]$ & $1.23[0]$ & $2.79[-1]$ & $4.61[-1]$ \\
13 & $3.55[-1]$ & $8.36[-1]$ & $4.46[-1]$ & $9.27[-1]$ & $1.86[-1]$ & $3.05[-1]$ \\
17 & $2.70[-1]$ & $6.46[-1]$ & $3.45[-1]$ & $7.22[-1]$ & $1.29[-1]$ & $2.08[-1]$ \\
21 & $2.06[-1]$ & $4.85[-1]$ & $2.80[-1]$ & $5.80[-1]$ & $9.26[-2]$ & $1.48[-1]$ \\
25 & $1.59[-1]$ & $3.83[-1]$ & $2.26[-1]$ & $4.74[-1]$ & $6.64[-2]$ & $1.09[-1]$ \\
29 & $1.28[-1]$ & $3.07[-1]$ & $1.85[-1]$ & $4.01[-1]$ & $5.16[-2]$ & $9.39[-2]$ \\
33 & $1.08[-1]$ & $2.71[-1]$ & $1.57[-1]$ & $3.54[-1]$ & $4.98[-2]$ & $8.73[-2]$ \\
37 & $9.82[-2]$ & $2.60[-1]$ & $1.46[-1]$ & $3.35[-1]$ & $3.90[-2]$ & $7.07[-2]$ \\
41 & $8.61[-2]$ & $2.20[-1]$ & $1.32[-1]$ & $2.94[-1]$ & $2.82[-2]$ & $4.51[-2]$ \\
60 & $3.65[-2]$ & $9.08[-2]$ & $8.13[-2]$ & $1.67[-1]$ & $1.14[-2]$ & $1.69[-2]$ \\
80 & $2.07[-2]$ & $4.94[-2]$ & $5.89[-2]$ & $1.12[-1]$ & $4.71[-3]$ & $6.66[-3]$ \\
100 & $1.33[-2]$ & $3.28[-2]$ & $4.23[-2]$ & $7.59[-2]$ & $2.55[-3]$ & $3.57[-3]$ \\
120 & $8.82[-3]$ & $2.09[-2]$ & $3.13[-2]$ & $5.51[-2]$ & $1.47[-3]$ & $1.97[-3]$ \\
140 & $6.91[-3]$ & $1.62[-2]$ & $2.50[-2]$ & $4.12[-2]$ & $8.10[-4]$ & $1.09[-3]$ \\
160 & $4.97[-3]$ & $1.13[-2]$ & $2.02[-2]$ & $3.16[-2]$ & $5.71[-4]$ & $7.20[-4]$ \\
200 & $2.79[-3]$ & $6.67[-3]$ & $1.24[-2]$ & $1.83[-2]$ & $2.30[-4]$ & $2.69[-4]$ \\
240 & $1.88[-3]$ & $4.18[-3]$ & $8.47[-3]$ & & $1.29[-4]$ & $1.33[-4]$ \\
280 & $1.24[-3]$ & $2.63[-3]$ & $5.42[-3]$ & & $7.76[-5]$ & $8.46[-5]$ \\
320 & $9.62[-4]$ & $2.00[-3]$ & $3.74[-3]$ & & $5.20[-5]$ & $4.72[-3]$ \\
360 & $7.17[-4]$ & $1.28[-3]$ & $2.50[-3]$ & & $3.21[-5]$ & $2.73[-5]$ \\
& & & & & &
\end{tabular}

The paper is organized as follows. In Sec. II, specifications of the experimental setup and the possible uncertainties in the measurements are described in brief. Section III describes the methodology to obtain the DDCS within the CDW-EIS framework. The discussion of the theoretical and experimental results and the comparison between them in terms of DDCS energy distributions at different angles are drawn in Sec. IV A. In Sec. IV B, the energy distributions of the DDCS ratio are discussed for different dressed projectile charge states to highlight the dressed projectile effect as a function of electron energy. Finally, Sec. V presents a summary of this work. In addition to that, the experimentally obtained absolute DDCS values for different charge states, at different angles, are tabulated in Tables II and III for He and Ne, respectively.

\section{EXPERIMENTAL DETAILS}

In the present experiment, we have measured the energy and angular distributions of the electron emission from $\mathrm{He}$ and $\mathrm{Ne}$ in collisions with $3.75 \mathrm{MeV} / \mathrm{u} \mathrm{O}^{q+}$ ions $(q=5,6,7,8)$. The ions were obtained from the BARC-TIFR $14 \mathrm{MV}$ tandem Pelletron accelerator facility in Mumbai, India. A well-collimated charge-state analyzed beam of $\mathrm{O}$ ions was made incident on the gas targets in a high vacuum chamber, where the base pressure was better than $2 \times 10^{-7}$ Torr. An electron spectrometer having $6 \%$ energy resolution, consisting of a hemispherical electrostatic analyzer and channel electron multiplier (CEM) detector, was used for electron detection [61]. The energy and angular distributions of the absolute 
TABLE III. Measured double-differential cross sections for $\mathrm{Ne}$ in units of $\mathrm{Mb} /(\mathrm{eV}$ sr) at different angles, for different charge states. For errors, see the text. Numerals in square brackets denote the power of 10 .

\begin{tabular}{|c|c|c|c|c|c|c|c|c|c|c|c|c|}
\hline$\epsilon_{e}(\mathrm{eV})$ & $\begin{array}{l}30^{\circ} \\
\mathrm{O}^{5+}\end{array}$ & $\begin{array}{l}30^{\circ} \\
\mathrm{O}^{6+}\end{array}$ & $\begin{array}{l}30^{\circ} \\
\mathrm{O}^{7+}\end{array}$ & $\begin{array}{l}30^{\circ} \\
\mathrm{O}^{8+}\end{array}$ & $\begin{array}{l}90^{\circ} \\
\mathrm{O}^{5+}\end{array}$ & $\begin{array}{l}90^{\circ} \\
\mathrm{O}^{6+}\end{array}$ & $\begin{array}{l}90^{\circ} \\
\mathrm{O}^{7+}\end{array}$ & $\begin{array}{l}90^{\circ} \\
\mathrm{O}^{8+}\end{array}$ & $\begin{array}{l}150^{\circ} \\
\mathrm{O}^{5+}\end{array}$ & $\begin{array}{l}150^{\circ} \\
\mathrm{O}^{6+}\end{array}$ & $\begin{array}{l}150^{\circ} \\
\mathrm{O}^{7+}\end{array}$ & $\begin{array}{l}150^{\circ} \\
\mathrm{O}^{8+}\end{array}$ \\
\hline 11 & $1.60[0]$ & [0] & [0] & & ] & [0] & ] & ] & 1. & $1.33[0]$ & 1.5 & $1.87[0]$ \\
\hline 13 & [0] & $0]$ & [0] & 0] & & & & & $3[-1]$ & {$[0]$} & & 0] \\
\hline 17 & $1.05[0]$ & $34[0]$ & $1.69[0]$ & $7[0]$ & $1.10[0]$ & $0[0]$ & 67[0] & $2.06[0]$ & $0[-1]$ & $9.22[-1]$ & $1.06[0]$ & $27[0]$ \\
\hline 21 & $8.82[-1]$ & $1.09[0]$ & $1.41[0]$ & $1.79[0]$ & $9.33[-1]$ & $1.06[0]$ & $1.38[0]$ & $1.72[0]$ & $5.86[-1]$ & $7.2[-1]$ & $8.30[-1]$ & $9.90[-1]$ \\
\hline 25 & $6.94[-1]$ & $8.95[-1]$ & $1.17[0]$ & $1.48[0]$ & $8.01[-1]$ & $9.14[-1]$ & $1.18[0]$ & $1.48[0]$ & $4.72[-1]$ & $5.78[-1]$ & $6.47[-1]$ & $7.92[-1]$ \\
\hline 29 & $6.07[-1]$ & $7.47[-1]$ & $9.85[-1]$ & $1.26[0]$ & $89[-1]$ & $7.76[-1]$ & $1.02[0]$ & $1.25[0]$ & $3.90[-1]$ & $4.51[-1]$ & $5.22[-1]$ & $6.11[-1]$ \\
\hline 33 & $5.35[-1]$ & $6.54[-1]$ & $8.56[-1]$ & $1.10[0]$ & $5.95[-1]$ & $6.77[-1]$ & $8.91[-1]$ & $1.09[0]$ & $3.04[-1]$ & $3.73[-1]$ & $4.39[-1]$ & $5.19[-1]$ \\
\hline 37 & $4.45[-1]$ & $5.67[-1]$ & $7.38[-1]$ & $9.35[-1]$ & $5.29[-1]$ & $5.90[-1]$ & $7.83[-1]$ & $9.61[-1]$ & $2.51[-1]$ & $3.00[-1]$ & $3.49[-1]$ & $4.13[-1]$ \\
\hline 41 & $3.84[-1]$ & $4.93[-1]$ & $6.31[-1]$ & $8.16[-1]$ & $4.75[-1]$ & $5.19[-1]$ & $6.87[-1]$ & $8.53[-1]$ & $2.17[-1]$ & $2.61[-1]$ & $2.89[-1]$ & $3.58[-1]$ \\
\hline 60 & $2.55[-1]$ & $3.15[-1]$ & $4.15[-1]$ & $5.32[-1]$ & $2.74[-1]$ & $3.05[-1]$ & $3.96[-1]$ & $4.89[-1]$ & $1.08[-1]$ & $1.28[-1]$ & $1.35[-1]$ & $1.65[-1]$ \\
\hline 80 & $1.71[-1]$ & $2.06[-1]$ & $2.74[-1]$ & $3.50[-1]$ & $1.88[-1]$ & $2.08[-1]$ & $2.65[-1]$ & $3.32[-1]$ & $5.78[-2]$ & $6.05[-2]$ & $6.64[-2]$ & $7.64[-2]$ \\
\hline 100 & $1.18[-1]$ & $1.40[-1]$ & $1.88[-1]$ & $2.37[-1]$ & $1.41[-1]$ & $1.49[-1]$ & $1.89[-1]$ & $2.39[-1]$ & $3.35[-2]$ & $3.53[-2]$ & $3.72[-2]$ & $4.37[-2]$ \\
\hline 120 & $7.95[-2]$ & $9.38[-2]$ & $1.23[-1]$ & $1.59[-1]$ & $1.09[-1]$ & $1.15[-1]$ & $1.42[-1]$ & $1.79[-1]$ & $1.95[-2]$ & $1.96[-2]$ & $2.00[-2]$ & $2.29[-2]$ \\
\hline 140 & $6.10[-2]$ & $7.20[-2]$ & $9.47[-2]$ & $1.19[-1]$ & $8.27[-2]$ & $8.33[-2]$ & $1.04[-1]$ & $1.28[-$ & $1.17[-2]$ & $1.30[-2]$ & $1.35[-2]$ & $1.51[-2]$ \\
\hline 160 & $4.97[-2]$ & $5.78[-2]$ & $7.59[-2]$ & $9.74[-2]$ & $6.99[-2]$ & $7.02[-2]$ & $8.51[-2]$ & $1.06[-1]$ & $8.73[-3]$ & $8.87[-3]$ & $8.89[-3]$ & $9.91[-3]$ \\
\hline 200 & $3.24[-2]$ & $3.79[-2]$ & $4.98[-2]$ & $6.25[-2]$ & $4.42[-2]$ & $4.31[-2]$ & $5.13[-2]$ & $6.39[-2]$ & $4.86[-3]$ & $4.56[-3]$ & $4.13[-3]$ & $4.94[-3]$ \\
\hline 240 & $2.27[-2]$ & $2.65[-2]$ & $3.34[-2]$ & $4.18[-2]$ & $3.37[-2]$ & $3.11[-2]$ & $3.78[-2]$ & $4.61[-2]$ & $3.10[-3]$ & $2.86[-3]$ & $2.66[-3]$ & $3.03[-3]$ \\
\hline 280 & $1.74[-2]$ & $1.93[-2]$ & $2.39[-2]$ & $3.02[-2]$ & $2.47[-2]$ & $2.20[-2]$ & $2.63[-2]$ & $3.22[-2]$ & $1.98[-3]$ & $1.77[-3]$ & $1.59[-3]$ & $1.70[-3]$ \\
\hline 320 & $1.29[-2]$ & $1.44[-2]$ & $1.74[-2]$ & $2.12[-2]$ & $1.93[-2]$ & $1.65[-2]$ & $1.93[-2]$ & $2.40[-2]$ & $1.38[-3]$ & $1.19[-3]$ & $1.11[-3]$ & $1.13[-3]$ \\
\hline 360 & $1.09[-2]$ & $1.17[-2]$ & $1.35[-2]$ & $1.71[-2]$ & $1.53[-2]$ & $1.32[-2]$ & $1.45[-2]$ & $1.78[-2]$ & $7.47[-4]$ & $7.42[-4]$ & $7.31[-4]$ & $7.42[-4]$ \\
\hline 400 & $8.32[-3]$ & $9.19[-3]$ & $1.09[-2]$ & $1.33[-2]$ & $1.28[-2]$ & $1.06[-2]$ & $1.14[-2]$ & $1.37[-2]$ & $6.47[-4]$ & $5.37[-4]$ & $5.14[-4]$ & $4.66[-4]$ \\
\hline
\end{tabular}

electron DDCS were studied for the emission angles $30^{\circ}, 90^{\circ}$, and $150^{\circ}$, in the energy range of 1 to $400 \mathrm{eV}$. The maximum statistical uncertainty, in the data, was about $15 \%$. Other than that, the uncertainty from gas pressure, which was about $7 \%$, contributes mainly to the absolute error. Further details of the experimental technique used are available in the preceding paper [62].

\section{THEORETICAL MODEL}

For the theoretical treatment, we have used the extension of the prior version of the CDW-EIS model for single ionization by dressed projectile impact, introduced by Monti et al. [52,53]. In those works, it has been shown that for the case of dressed projectiles, it is not enough to describe the projectile potential as a pure Coulomb one given by the projectile net charge. This description corresponds to considering the projectile to be completely screened by its electrons. Therefore, in the extension presented in [52,53], the projectile potential $V_{P}$ is approximated by a parametric GSZ potential [48]. This potential presents a short-range term and a Coulomb long-range term depending on the net charge $q$ of the projectile,

$$
V_{P}(s)=-\frac{1}{s}\left(Z_{P}-q\right)\left[H\left(e^{s / d}-1\right)+1\right]^{-1}-\frac{q}{s} .
$$

In (1), $H$ and $d$ are the parameters that shape the shortrange term and depend on the nuclear charge $Z_{P}$ of the projectile and the number of electrons $N_{e}$ bound to it. Also, $s$ is the distance between the target active electron and the projectile nucleus. In [53], it is shown that the long-range term is the main interaction leading to the ionization for large impact parameters; in this case, the projectile electrons only intervene screening the projectile nuclear charge. However, as the impact parameter reduces, the active electron interacts with the projectile electrons as well as the nucleus, and thus the short-range term dominates the ionization mechanism for short enough impact parameters. In the CDW-EIS framework, the initial- and the final-state wave functions of the target active electron are distorted by an eikonal phase and a confluent hypergeometric function, respectively, in which the projectile is asymptotically represented by its asymptotic net charge $q$. It has been shown in [63] that when the projectile degree of ionization $(q)$ is low enough, the projectile electrons are not bound enough to be considered as passive and therefore can also be ionized. Furthermore, also the simultaneous ionization can give a non-negligible contribution to the total electron emission. Thus, in order to calculate the contribution to electron emission given by the projectile ionization, the collision is reversed and the corresponding cross sections for projectile ionization are calculated in a projectile fixed reference frame and then transformed to the laboratory frame by the well-known expression

$$
\frac{d \sigma(\theta, \varepsilon)}{d \Omega d \varepsilon}=\left(\frac{\varepsilon}{\varepsilon^{\prime}}\right)^{1 / 2} \frac{d \sigma^{\prime}\left(\theta^{\prime}, \varepsilon^{\prime}\right)}{d \Omega^{\prime} d \varepsilon^{\prime}},
$$

where the primed (unprimed) quantities are associated with the projectile (laboratory) rest frame (see, for example, Ref. [1]). The simultaneous ionization is then estimated by a simple probabilistic approach weighting the DDCS by the TCS of target and projectile ionization, as previously used in [63]:

$$
\operatorname{DDCS}^{(\operatorname{sim})}=\frac{\operatorname{DDCS}^{(T)} \operatorname{TCS}^{(P)}}{\operatorname{TCS}^{(T)}+\operatorname{TCS}^{(P)}}+\frac{\operatorname{DDCS}^{(P)} \operatorname{TCS}^{(T)}}{\operatorname{TCS}^{(T)}+\operatorname{TCS}^{(P)}} .
$$

In Eq. (3), $\operatorname{DDCS}^{(T, P)}$ and $\operatorname{TCS}^{(T, P)}$ stand for the DDCS and total cross sections (TCS) for target ionization and 


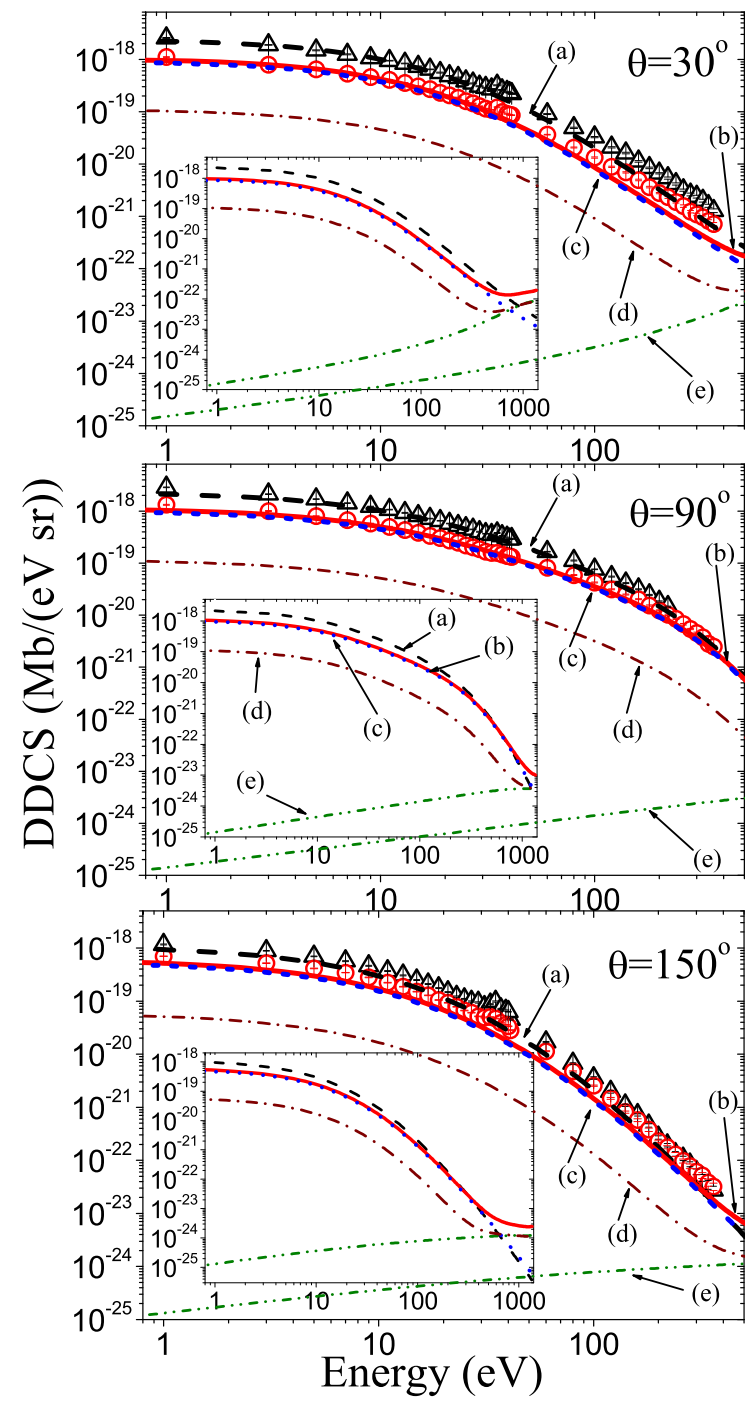

FIG. 1. (Color online) DDCS energy distributions for He target at $30^{\circ}, 90^{\circ}$, and $150^{\circ}$. DDCS corresponding to $\mathrm{O}^{8+}\left(\mathrm{O}^{5+}\right)$ is symbolized by a black triangle (red circle). CDW-EIS calculations are denoted as follows: (a) Black dashed line: DDCS corresponding to $\mathrm{O}^{8+}$; (b) red solid line: DDCS corresponding to $\mathrm{O}^{5+}$; (c) blue dotted line: target ionization; (d) brown dash-dotted line: target-projectile simultaneous ionization; (e) green dash-double-dotted line: projectile ionization. Inset: Same CDW-EIS plots extended up to higher electron energy.

projectile electron loss, respectively, at the given projectile velocity $v$. This approach can give a rough estimation of the simultaneous ionization contribution and does not take into account any interference effects coming from target and projectile ionization.

\section{RESULTS AND DISCUSSIONS}

\section{A. DDCS energy distribution}

The electron DDCS spectra as a function of electron energy, at $30^{\circ}, 90^{\circ}$, and $150^{\circ}$, are shown for the He target, along with the CDW-EIS predictions in Fig. 1. Similar spectra for the $\mathrm{Ne}$ target are displayed in Fig. 2. In each plot, the DDCS corresponding to the $\mathrm{O}^{5+}$ projectile (red circle) has been

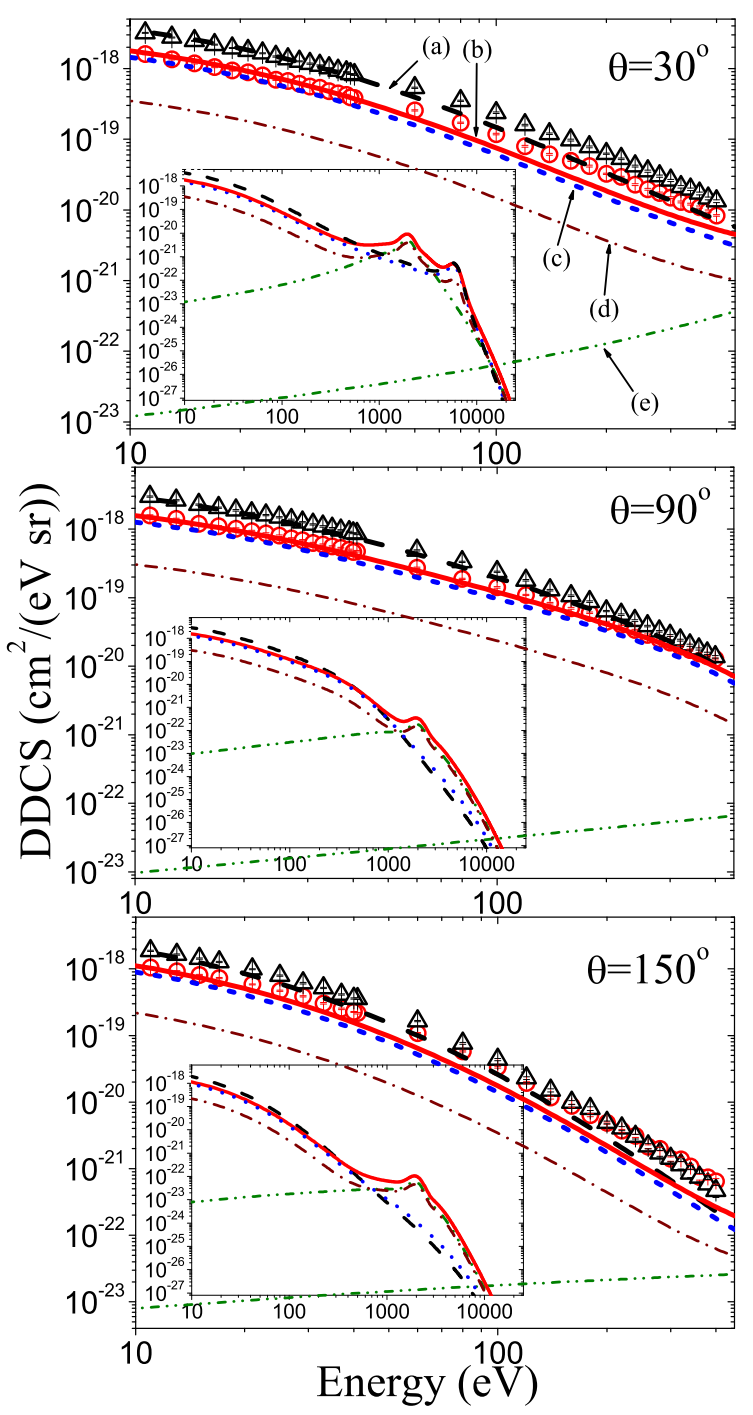

FIG. 2. (Color online) Same as Fig. 1 for Ne target.

compared to that for the $\mathrm{O}^{8+}$ (black triangle). The CDW-EIS calculation for the $\mathrm{O}^{8+}$ impact has been denoted by a black dash line (a). In the case of the dressed ion impact, different ionization processes can contribute to the measured electron spectra. By means of the CDW-EIS calculations, in addition to the total DDCS [(b): red solid line], we have estimated the individual contributions from target ionization [(c): blue dotted line], target-projectile simultaneous ionization [(d): brown dash-dotted line], and projectile ionization [(e): green dash-double-dotted line]. The experimental data have been taken between 1 to $400 \mathrm{eV}$, whereas the theoretical calculations are extended to $1500 \mathrm{eV}$ (inset in Fig. 1) for $\mathrm{He}$ and to $2500 \mathrm{eV}$ (inset in Fig. 2) for Ne.

In general, the DDCS data show a quick fall with increasing electron energy for both of the charge states in accordance with the common trend in the case of ion-atom collisions. In the soft collision region $(\leqslant 30 \mathrm{eV})$, for both the targets, the DDCS corresponding to $\mathrm{O}^{8+}$ is higher than that for $\mathrm{O}^{5+}$. However, with increasing energy, these two cross sections seem to come closer to each other. In fact, at some value of energy, DDCS for the $\mathrm{O}^{5+}$ projectile crosses over that for the $\mathrm{O}^{8+}$. This 
crossover value varies with the observation angle. For example, in Fig. 1, at $30^{\circ}$ and $90^{\circ}$, it is around $630 \mathrm{eV}$, as shown by the CDW-EIS calculations (experimental data is present up to less energy), while at $150^{\circ}$, it is around $310 \mathrm{eV}$, obtained from both the experiment and the theory. After this crossover, the bare projectile cross section goes below the dressed projectile cross section.

As far as absolute agreement between the experiment and the calculation is concerned, in the case of He (Fig. 1), it is excellent throughout the whole energy range for $90^{\circ}$, and in the low-energy (up to $60 \mathrm{eV}$ ) region for the rest of the angles, for both of the charge states. In the high-energy region, at these two remaining angles, the theory starts underestimating the data to some extent (by a factor of 2-3). It should be mentioned here that the little humplike structure at $35 \mathrm{eV}$ is due to the autoionization of the doubly excited state of He, and the theory does not take into account such characteristic excitation process. But if we look at the overall qualitative agreement, it is quite good for both of the charge states, for all angles. In the case of $\mathrm{Ne}$ (Fig. 2), the situation is quite similar, though the deviations at extreme angles start a little earlier from about $35 \mathrm{eV}$, and in the high-energy region the deviation is little more than that of He. A comparison between two targets suggests that the overall agreement is better in the case of the He than that for the $\mathrm{Ne}$.

The behavior of the dressed projectile impact DDCS spectrum can be understood much more clearly if we look at the individual contributions from different ionization processes. From very low energy to the crossover point, target ionization (c) is the most dominant process and it follows a decreasing trend as in case of the bare ion-atom collision. Simultaneous ionization (d) is also showing the same qualitative behavior as the previous one. But it has less contribution to the total DDCS. On the other hand, the qualitative behavior of the projectile ionization (e) is completely different. It increases with the ejected electron energy. After the crossover point, simultaneous ionization (d) and projectile ionization (e) dominate over the target ionization (c). The dominance of the projectile electron loss in the high-energy region, especially at forward angles, was experimentally shown earlier by Stolterfoht et al. [39]. A comparison among different angles suggests that at intermediate and backward angles, simultaneous ionization (d) and projectile ionization (e) contributions are relatively smaller compared to the case of the forward angle, particularly up to the crossover energy. After that, only these two start dominating over the target ionization.

In the case of $\mathrm{Ne}$ (Fig. 2), the inset plots clearly indicate the electron loss peak at around $2 \mathrm{keV}$ at all three angles. The BE peak, as expected, is also visible at around $6 \mathrm{keV}$ for both the bare and the dressed projectiles (clearly visible at $30^{\circ}$ ). The other overall qualitative features are quite similar to that of the $\mathrm{He}$, though the absolute values of the contributions can differ between the targets.

\section{B. DDCS ratio}

To get a clearer picture of the dressed projectile effect, we have divided the DDCS corresponding to the $\mathrm{O}^{q+}$ projectile by that of the bare ion projectile [i.e., DDCS $\left(\mathrm{O}^{q+}\right) / \mathrm{DDCS}$ $\left(\mathrm{O}^{8+}\right)$ ]. In Fig. 3, the ratios for $q=5$, for the He target, are plotted as a function of electron energy for three different angles (i.e., $30^{\circ}, 90^{\circ}, 150^{\circ}$ ) and the corresponding CDW-EIS predictions are represented by the red solid line $(\mathrm{m})$. From the plots, it is evident that the overall agreement between the theoretical (CDW-EIS) predictions $(\mathrm{m})$ and the experimental data is quite well throughout the whole energy range for all angles. The characteristic dip at around $35 \mathrm{eV}$ is not taken into account in the theory, as mentioned earlier.

Low-energy electrons are produced in large impact parameter collisions, in which the projectile nucleus, as observed by the active electron, is expected to be screened by its residual electrons. In earlier measurements in the low-energy region, the results have been explained in terms of the Born scaling [40,44], i.e., the DDCS follows the $q^{2}$ scaling. Accordingly, the DDCS ratio should be $5^{2} / 8^{2}$ (i.e., 0.39), which is marked by the blue dashed line (n) in each plot of the figure (Fig. 3). But the present measurement shows a clear deviation from the $q^{2}$ scaling throughout the entire energy range. For all angles, the ratio values are higher than the $q^{2}$ value. Moreover, the amount of deviation at a fixed energy is also observed to be angle

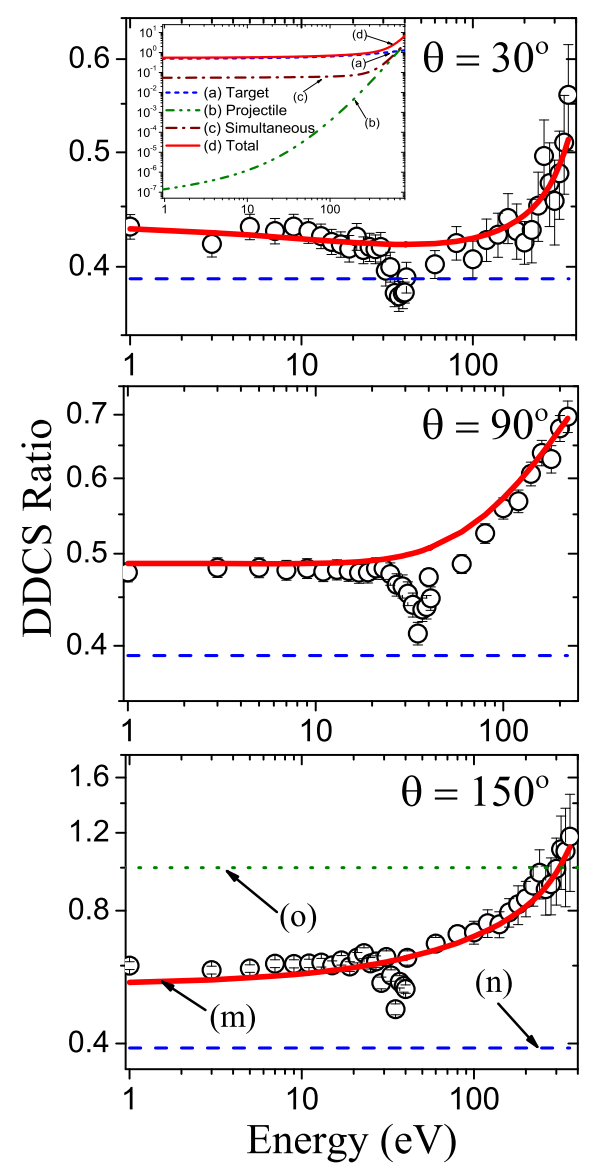

FIG. 3. (Color online) Energy distribution of DDCS ratio $\left(\mathrm{O}^{5+} / \mathrm{O}^{8+}\right)$ at different angles for the He target. The CDW-EIS calculation is denoted by a red solid line $(\mathrm{m})$, which is the ratio of total DDCS corresponding to $\mathrm{O}^{5+}$ and $\mathrm{O}^{8+}$. The blue dashed line $(\mathrm{n})$ denotes the Born $\left(q^{2}\right)$ prediction. The green dotted line (o) (in $150^{\circ}$ plot) indicates the crossover point, i.e., ratio $=1.0$. Inset: Contributions from different ionization processes to the total DDCS ratio. 
dependent. For example, at $30^{\circ}$, the experimentally measured ratio at the lowest energy $(1 \mathrm{eV})$ is about $10 \%$ higher than the expected $q^{2}$ value, whereas it is about $23 \%$ and $50 \%$ higher for $90^{\circ}$ and $150^{\circ}$, respectively.

The deviation from $Z_{p}^{2}$ scaling, in the case of highly charged bare ions, has been reported earlier [15-17]. It was quite successfully explained by the CDW-EIS model incorporating the projectile postcollision effect. This is also called the two-center effect (TCE). In the postcollision situation, the active electron is affected by the asymptotic Coulomb charge of the projectile. The projectile Coulomb drag leads to an enhancement in the cross section in the forward direction and a decrease in the backward direction. This causes additional forward-backward angular asymmetry in the DDCS angular distribution. This asymmetry increases with charge state because of the change in the strength of the projectile field. As a result, at the forward and the backward angles, the deviations are in opposite direction with respect to the $q^{2}$ predicted value and it increases with charge states. This was confirmed by the above-mentioned references (for example, see Fig. 5.23 on p. 134 and Figs. 5.24a and 5.24b on p. 135 in Ref. [1]). But in the present case, for all angles, the ratio values are at the higher side of the $q^{2}$-predicted value $\left(q^{2} / Z_{p}^{2}\right)$, i.e., the deviations are in the same direction.

We have confirmed these observations by doing a separate experiment with the $\mathrm{Ne}$ target and with a greater number of projectile charge states: $q=5,6,7,8$. In Fig. 4, the DDCS ratios, similar to Fig. 3, are plotted for $q=5,6$, and 7 . These plots also clearly show the unidirectional deviation. In this case, if the deviation is only caused by the TCE, at $30^{\circ}$, we would expect the lower values of ratios than the $q^{2}$-predicted value [blue dashed line (n)]. This is because the enhancement in the DDCS, at this angle, due to the TCE, is more for the $\mathrm{O}^{8+}$ than the $\mathrm{O}^{q+} \mathrm{s}$. The opposite behavior, observed here, can be explained if the DDCS corresponding to the dressed ion projectiles intrinsically do not follow the $q^{2}$ dependence. It may happen that the influence of the projectile electrons (so-called antiscreening) enhances the DDCS with respect to the expected $q^{2}$ scaled DDCS even at large impact parameter collisions for all angles. Then the ratio values would be greater than the $q^{2}$-predicted value at the forward angle. At the backward angle, TCE adds to this deviation and makes the ratio values much greater than the $q^{2}$ predicted value. This picture is also supported by the fact that with lower $q$ value, i.e., with more projectile electrons, the deviation is greater. For example, at $30^{\circ}$ and at $11 \mathrm{eV}, \mathrm{O}^{5+}$ data deviates by about $29 \%$, whereas the deviations are $10 \%$ and $2 \%$ in the cases of $\mathrm{O}^{6+}$ and $\mathrm{O}^{7+}$, respectively. These are about 44\%, 26\%, and 9\%, respectively, at $150^{\circ}$.

As far as the qualitative behavior of the ratio distribution is concerned, it remains nearly flat from the lowest energy until a certain higher energy, and then it rapidly increases to higher

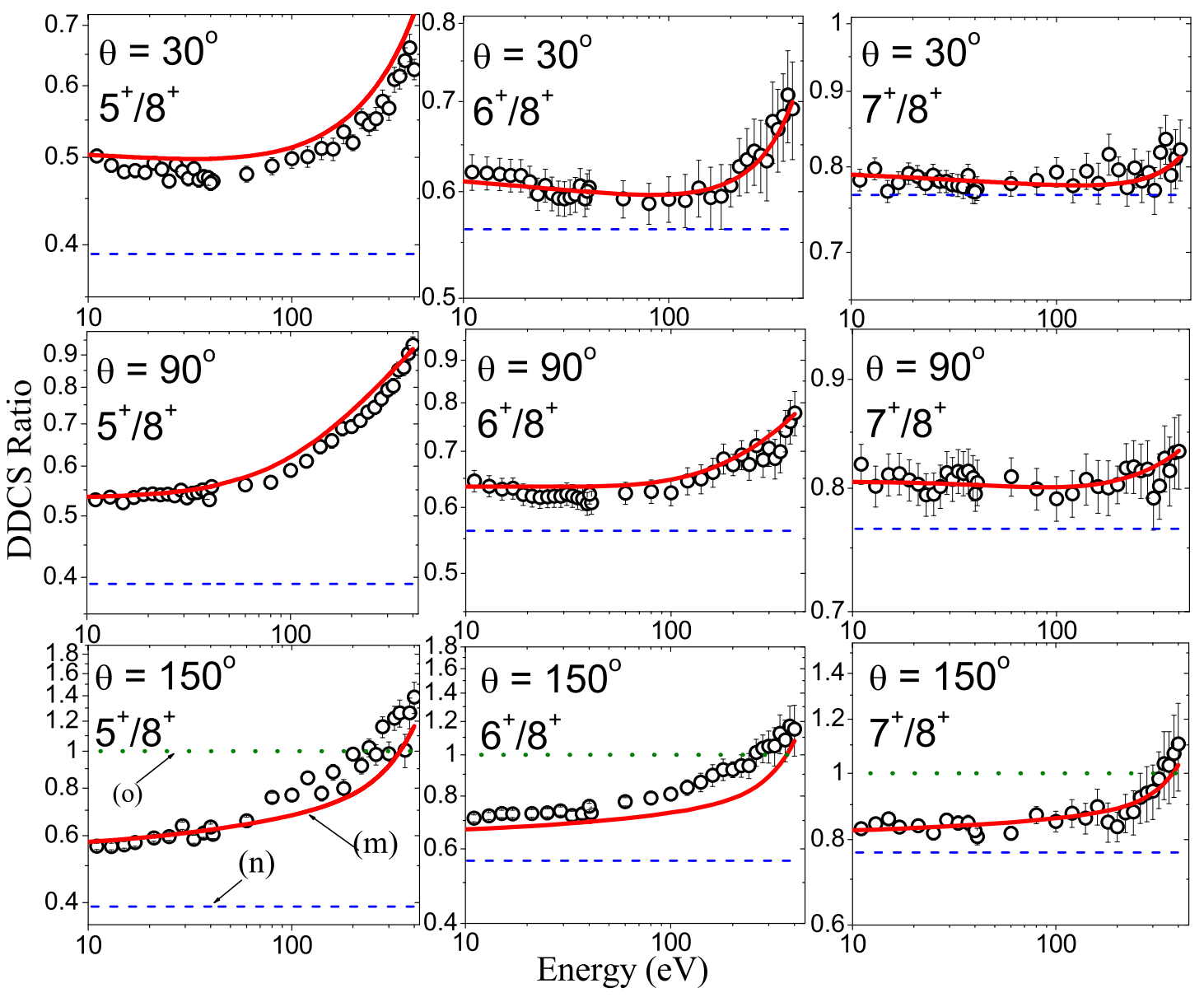

FIG. 4. (Color online) Same as Fig. 3 for Ne target for different $q$ values. 
values. Similar behavior, reported in Refs. [40] and [44], was explained in terms of the variation of projectile screening with the impact parameter of the collision. In the very low-energy part of the spectrum, where the target ionization dominates, the impact parameter of the collision remains much larger than the effective radius of the dressed ion. As a result, the active electron of the target observes the projectile nucleus and the remaining projectile electron cloud as a whole. The screened projectile of charge state $q$ would influence the active electron, until a certain impact parameter. Therefore, the ratio distribution remains nearly flat up to the corresponding energy. However, for the emission of higher-energy electrons (in low-impact parameter collisions), the distance between the active electron and the projectile nucleus is smaller than that involved in the lower-energy electron emission (in large-impact parameter collisions). Therefore, the electron interacts with a less screened projectile. This reduction of screening increases the effective charge to greater than $q$, which in turn increases the cross section rapidly, tending to approximate the cross section corresponding to $\mathrm{O}^{8+}$. We have also tried to understand this qualitative behavior, in more detail, by analyzing the individual contributions from different ionization processes. In the inset of Fig. 3, this is illustrated by means of the DDCS ratios. One can see that the target ionization cross section, indicated as (a), slowly increases with electron energy, which is compatible with the above-mentioned argument of the reduction of projectile screening. But apart from that, it also shows that although in the low-energy part only the target ionization governs the overall features, in the high-energy region projectile ionization (b) and simultaneous ionization (c) contribute considerably to the total electron emission. As a result of this projectile electron loss contribution, the overall DDCS ratio increases very rapidly after around $100 \mathrm{eV}$. In fact, it makes the DDCS ratio value greater than 1.0 (crossover point in Figs. 1 and 2), i.e., the total electron emission cross section for the dressed ion is greater than that for the bare ion (clearly visible in the case of $150^{\circ}$ ).

As discussed earlier, at the very low-energy part of the spectrum where target ionization is the dominant process, the target active electron observes the fully screened projectile, i.e., charge state $q$. As a result, the cross section is expected to vary as $q^{n}$. From the DDCS ratios [DDCS $\left.\left(\mathrm{O}^{q+}\right) / \operatorname{DDCS}\left(\mathrm{O}^{8+}\right)\right]$ corresponding to $q=5,6,7$ at $11 \mathrm{eV}$ for the Ne target, we have derived the value of $n$ for different angles. Both experimentally and theoretically (CDW-EIS) obtained values are displayed in Table I. It shows that the value of $n$ deviates more from 2.0 with the increasing value of observation angle. Now by using the values of $n$ and the DDCS ratio, the effective charge states seen by the active electrons of different energies can be determined. As an example, some representative values are displayed in Table IV, for the Ne target, where the initial charge state is $q=5$. A similar kind of analysis was reported earlier $[41,44]$, which, however, assumed a quadratic $q$ dependence. We find that for backward angles, the effective charge is much higher than $5+$, i.e., tending towards $8+$. This again indicates the higher degree of reduction in screening in the case of backward scattering.

If we compare between the two targets in terms of the absolute values of the DDCS ratios, we see that, in general,
TABLE IV. Values of effective charge states seen by the active electrons of different energies for the case of Ne target impacted by $\mathrm{O}^{5+}$. Typical error in deduced effective $q$ is about $5-10 \%$.

\begin{tabular}{lllr}
\hline \hline Energy $(\mathrm{eV})$ & $30^{\circ}$ & $90^{\circ}$ & $150^{\circ}$ \\
\hline 25 & 4.8 & 5.0 & 5.1 \\
60 & 4.8 & 5.1 & 5.4 \\
120 & 5.0 & 5.5 & 6.9 \\
200 & 5.2 & 6.0 & 7.4 \\
\hline \hline
\end{tabular}

the values are slightly higher in the case of $\mathrm{Ne}$. As a result, the $q$ dependence would be different for the He and the Ne. Therefore, the nature of the target also influences the results.

\section{CONCLUSION}

To summarize, we have measured the absolute DDCS of electron emission for ionization of the $\mathrm{He}$ and the $\mathrm{Ne}$ colliding with the dressed oxygen ionic projectiles. This has been compared with the DDCS for the bare ion projectiles at three different angles, ranging from the forward to the backward direction.

It is observed that the DDCS does not follow the $q^{2}$ scaling and the deviation starts right from very low emission energy. For large-impact parameter collisions where the active electron is supposed to see the fully screened dressed projectile charge state, the deviation is probably caused by the active influence of the projectile electrons on target ionization. It enhances the DDCS on top of the predicted- $q^{2}$ scaled DDCS. For the lowimpact parameter collisions, i.e., in the high-energy region of the DDCS spectrum, the reduction of the projectile screening with the reduction of the impact parameter causes the larger deviation. A higher degree of reduction in screening in the case of backward scattering is observed.

The present form of the CDW-EIS model shows a very good general agreement with the experimental data for both of the targets. It is realized that the GSZ potential is necessary to describe the influence of projectile electrons. The relative contributions from different ionization processes, relevant for the dressed ion impact, are also investigated theoretically. It suggests that in the low-energy part of the spectrum, target ionization is the dominant process, whereas with increasing electron energy, different projectile electron loss processes start contributing significantly. Even after certain energy, the target projectile simultaneous ionization and projectile ionization dominated over the target ionization. The analysis of both of the targets suggests that the $q$ dependence of the DDCS spectra also differs with the target species.

\section{ACKNOWLEDGMENTS}

The authors would like to thank the staffs at the BARCTIFR Pelletron accelerator facility for smooth operation of the machine. J.M.M. and R.D.R. acknowledge support from the Agencia Nacional de Promoción Científica y Tecnológica of Argentina through the Project No. PICT 2011-2145. 
[1] N. Stolterfoht, R. D. DuBois, and R. D. Rivarola, Electron Emission in Heavy Ion-Atom Collisions (Springer-Verlag, Berlin, 1997).

[2] Lokesh C. Tribedi, P. Richard, Y. D. Wang, C. D. Lin, and R. E. Olson, Phys. Rev. Lett. 77, 3767 (1996).

[3] N. Stolterfoht, B. Sulik, V. Hoffmann, B. Skogvall, J. Y. Chesnel, J. Rangama, F. Frémont, D. Hennecart, A. Cassimi, X. Husson, A. L. Landers, J. A. Tanis, M. E. Galassi, and R. D. Rivarola, Phys. Rev. Lett. 87, 023201 (2001).

[4] Deepankar Misra, U. Kadhane, Y. P. Singh, L. C. Tribedi, P. D. Fainstein, and P. Richard, Phys. Rev. Lett. 92, 153201 (2004).

[5] D. Misra, A. Kelkar, U. Kadhane, A. Kumar, L. C. Tribedi, and P. D. Fainstein, Phys. Rev. A 74, 060701(R) (2006).

[6] D. Misra, A. Kelkar, U. Kadhane, A. Kumar, Y. P. Singh, Lokesh C. Tribedi, and P. D. Fainstein, Phys. Rev. A 75, 052712 (2007).

[7] S. Nandi, A. N. Agnihotri, S. Kasthurirangan, A. Kumar, C. A. Tachino, R. D. Rivarola, F. Martín, and L. C. Tribedi, Phys. Rev. A 85, 062705 (2012).

[8] J. H. McGuire, N. Stolterfoht, and P. R. Simony, Phys. Rev. A 24, 97 (1981).

[9] O. Woitke, P. A. Závodszky, S. M. Ferguson, J. H. Houck, and J. A. Tanis, Phys. Rev. A 57, 2692 (1998).

[10] Chen Lin, Chen Xi-Meng, Shao Jian-Xiong, Lu Yan-Xia, Ding Bao-Wei, Cui Ying, Gao Zhi-Min, Liu Yu-Wen, Du Juan, Xie Jiang-Shan, Sun Guang-Zhi, and Liu Zhao-Yuan, Chin. Phys. 16, 2378 (2007).

[11] Wang Bao-Hong, Fu Hong-Bin, Ding Bao-Wei, Yu De-Yang, Sun Guang-Zhi, and Liu Zhao-Yuan, Chin. Phys. Lett. 25, 2460 (2008).

[12] Baowei Ding, Phys. Scr. 85, 015302 (2012).

[13] E. C. Montenegro, G. M. Sigaud, and R. D. DuBois, Phys. Rev. A 87, 012706 (2013).

[14] A. S. Schlachter, K. H. Berkner, W. G. Graham, R. V. Pyle, P. J. Schneider, K. R. Stalder, J. W. Stearns, J. A. Tanis, and R. E. Olson, Phys. Rev. A 23, 2331 (1981).

[15] Pablo D. Fainsteint, Victor H. Ponce, and Roberto D. Rivarola, J. Phys. B: At. Mol. Opt. Phys. 21, 287 (1988).

[16] J. O. P. Pedersent, P. Hvelplundt, A. G. Petersent, and P. D. Fainstein, J. Phys. B: At. Mol. Opt. Phys. 23, L597 (1990).

[17] Pablo D. Fainsteint, Victor H. Ponce, and Roberto D. Rivarola, J. Phys. B: At. Mol. Opt. Phys. 24, 3091 (1991).

[18] N. Stolterfoht, H. Platten, G. Schiwietz, D. Schneider, L. Gulyás, P. D. Fainstein, and A. Salin, Phys. Rev. A 52, 3796 (1995).

[19] U. Ramm, U. Bechthold, O. Jagutzki, S. Hagmann, G. Kraft, H. Schmidt-Böcking, Nucl. Instrum. Methods Phys. Res. B 98, 359 (1995).

[20] P. Richard, D. H. Lee, T. J. M. Zouros, J. M. Sanders, and J. L. Shinpaugh, J. Phys. B: At. Mol. Opt. Phys. 23, L213 (1990).

[21] R. E. Olson, C. O. Reinhold, and D. R. Schultz, J. Phys. B: At. Mol. Opt. Phys. 23, L455 (1990).

[22] R. Shingal, Z. Chen, K. R. Karim, C. D. Lin, and C. P. Bhalla, J. Phys. B: At. Mol. Opt. Phys. 23, L637 (1990).

[23] A. D. González, P. Dahl, P. Hvelplund, and K. Taulbjerg, J. Phys. B: At. Mol. Opt. Phys. 25, L573 (1992).

[24] T. J. M. Zouros, P. Richard, K. L. Wong, H. I. Hidmi, J. M. Sanders, C. Liao, S. Grabbe, and C. P. Bhalla, Phys. Rev. A 49, R3155(R) (1994).

[25] C. Liao, P. Richard, S. R. Grabbe, C. P. Bhalla, T. J. M. Zouros, and S. Hagmann, Phys. Rev. A 50, 1328 (1994).
[26] C. O. Reinhold, D. R. Schultz, R. E. Olson, C. Kelbch, R. Koch, and H. Schmidt-Böcking, Phys. Rev. Lett. 66, 1842 (1991).

[27] N. Stolterfoht, C. C. Havener, R. A. Phaneuf, J. K. Swenson, S. M. Shafroth, and F. W. Meyer, Phys. Rev. Lett. 57, 74 (1986).

[28] N. Stolterfoht, C. C. Havener, R. A. Phaneuf, J. K. Swenson, S. M. Shafroth, and F. W. Meyer, Phys. Rev. Lett. 58, 958 (1987).

[29] N. Stolterfoht, P. D. Miller, H. F. Krause, Y. Yamazaki, J. K. Swenson, R. Bruch, P. F. Dittner, P. L. Pepmiller, and S. Datz, Nucl. Instrum. Methods Phys. Res. B 24-25, 168 (1987).

[30] T. J. M. Zouros, D. H. Lee, and P. Richard, Phys. Rev. Lett. 62, 2261 (1989).

[31] H.-P. Hülskötter, W. E. Meyerhof, E. Dillard, and N. Guardala, Phys. Rev. Lett. 63, 1938 (1989).

[32] R. Dörner, V. Mergel, R. Ali, U. Buck, C. L. Cocke, K. Froschauer, O. Jagutzki, S. Lencinas, W. E. Meyerhof, S. Nüttgens, R. E. Olson, H. Schmidt-Böcking, L. Spielberger, K. Tökesi, J. Ullrich, M. Unverzagt, and W. Wu, Phys. Rev. Lett. 72, 3166 (1994).

[33] J. Wang, J. H. McGuire, and E. C. Montenegro, Phys. Rev. A 51, 504 (1995).

[34] D. R. Bates and G. W. Griffing, Proc. R. Phys. Soc. London A 66, 961 (1953).

[35] D. R. Bates and G. W. Griffing, Proc. R. Phys. Soc. London A 68, 90 (1955).

[36] N. Stolterfoht, Phys. Scr. 42, 192 (1990).

[37] N. Stolterfoht, Nucl. Instrum. Methods Phys. Res. B 53, 477 (1991)

[38] W. Wolff, H. Luna, A. C. F. Santos, E. C. Montenegro, R. D. DuBois, C. C. Montanari, and J. E. Miraglia, Phys. Rev. A 84, 042704 (2011).

[39] N. Stolterfoht, D. Schneider, D. Burch, H. Wieman, and J. S. Risley, Phys. Rev. Lett. 33, 59 (1974).

[40] N. Stolterfoht, Structure and Collisions of Ions and Atoms, Topics in Current Physics, Vol. 5, edited by I. A. Sellin (Springer-Verlag, Berlin, 1978), p. 155.

[41] L. H. Toburen, N. Stolterfoht, P. Ziem, and D. Schneider, Phys. Rev. A 24, 1741 (1981)

[42] J. Schader, R. Latz, M. Burkhard, H. J. Frischkorn, D. Hofmann, P. Koschar, K. O. Groeneveld, D. Bernyi, A. Kövér, and Gy. Szabó, J. Phys. Lett. 45, 249 (1984).

[43] R. D. DuBois, L. H. Toburen, M. E. Middendorf, and O. Jagutzki, Phys. Rev. A 49, 350 (1994).

[44] N. Stolterfoht, D. Schneider, D. Burch, H. Wieman, and J. S. Risley, Phys. Rev. A 49, 5112 (1994).

[45] L. H. Toburen and R. D. DuBois, Radiat. Prot. Dosim. 52, 129 (1994).

[46] M. Kuzel, O. Heil, R. Maier, M. W. Lucas, D. H. JakubassaAmundsen, B. W. Farmery, and K. O. Groeneveld, J. Phys. B: At. Mol. Opt. Phys. 25, 1839 (1992).

[47] O. Heil, R. D. DuBois, R. Maier, M. Kuzel, and K. O. Groeneveld, Z. Phys. D 21, 235 (1991).

[48] A. E. S. Green, D. L. Sellin, and A. S. Zachor, Phys. Rev. 184, 1 (1969).

[49] J. M. Monti, O. A. Fojón, J. Hanssen, and R. D. Rivarola, J. Phys. B: At. Mol. Opt. Phys. 46, 145201 (2013).

[50] D. S. F. Crothers and J. F. McCann, J. Phys. B: At. Mol. Opt. Phys. 16, 3229 (1983).

[51] L. Gulyás, P. D. Fainstein, and A. Salin, J. Phys. B: At. Mol. Opt. Phys. 28, 245 (1995). 
[52] J. M. Monti, R. D. Rivarola, and P. D. Fainstein, J. Phys. B: At. Mol. Opt. Phys. 41, 201001 (2008).

[53] J. M. Monti, R. D. Rivarola, and P. D. Fainstein, J. Phys. B: At. Mol. Opt. Phys. 44, 195206 (2011).

[54] F. Drepper and J. S. Briggs, J. Phys. B: At. Mol. Opt. Phys. 9, 2063 (1976).

[55] Steven T. Manson and L. H. Toburen, Phys. Rev. Lett. 46, 529 (1981).

[56] R. D. DuBois and Steven T. Manson, Phys. Rev. Lett. 57, 1130 (1986).

[57] Helene M. Hartley and H. R. J. Walters, J. Phys. B: At. Mol. Opt. Phys. 20, 3811 (1987).

[58] Á. Kövér, G. Szabó, L. Gulyás, K. Tökési, D. Berényi, O. Heil, and K. O. Groeneveld, J. Phys. B: At. Mol. Opt. Phys. 21, 3231 (1988).
[59] Jianyi Wang, Carlos O. Reinhold, and Joachim Burgdörfer, Phys. Rev. A 45, 4507 (1992).

[60] D. Schneider, M. Prost, N. Stolterfoht, G. Nolte, and R. Du Bois, Phys. Rev. A 28, 649 (1983).

[61] D. Misra, K. V. Thulasiram, W. Fernandes, A. H. Kelkar, U. Kadhane, A. Kumar, Y. P. Singh, L. Gulyas, and L. C. Tribedi, Nucl. Instrum. Methods Phys. Res. B 267, 157 (2009).

[62] Shubhadeep Biswas, D. Misra, J. M. Monti, C. A. Tachino, R. D. Rivarola, and L. C. Tribedi, Phys. Rev. A 90, 052714 (2014).

[63] J. M. Monti, J. Fiol, D. Fregenal, P. D. Fainstein, R. D. Rivarola, W. Wolff, E. Horsdal, G. Bernardi, and S. Suáarez, Phys. Scr. T156, 014031 (2013). 